\title{
AVERAGE KISSING NUMBERS FOR NON-CONGRUENT SPHERE PACKINGS
}

\author{
Greg Kuperberg and Oded Schramm
}

\section{Introduction}

Let $P$ be a packing of $n$ (round) balls in $\mathbb{R}^{3}$. (A packing of round balls, also known as a sphere packing, is a collection of round balls with disjoint interiors.) The balls may have different radii. The average kissing number of $P$ is defined as $k(P)=2 m / n$, where $m$ is the number of tangencies between balls in the packing. Let

$$
k=\sup \left\{k(P) \mid P \text { is a finite packing of balls in } \mathbb{R}^{3}\right\} .
$$

\section{Theorem 1.}

$$
12.566 \approx 666 / 53 \leq k<8+4 \sqrt{3} \approx 14.928
$$

(The appearance of the number of the beast in the lower bound is purely coincidental.)

The supremal average kissing number $k$ is defined in any dimension, as are $k_{c}$, the supremal average kissing number for congruent ball packing, and $k_{s}$, the maximal kissing number for a single ball surrounded by congruent balls with disjoint interiors. (Clearly, $k_{c} \leq k$ and $k_{c} \leq k_{s}$.) It is interesting that $k$ is always finite, because a large ball can be surrounded by many small balls in a non-congruent ball packing. Nevertheless, a simple argument presented below shows that $k \leq 2 k_{s}$ in every dimension, and clearly $k_{s}$ is always finite. In two dimensions, an Euler characteristic argument shows that $k \leq 6$, but it is also well-known that $k_{s}=k_{c}=6$. One might therefore conjecture that $k=k_{c}$ always, or at least in dimensions such as $2,3,8$, and 24 (and conjecturally several others) in which $k_{s}=k_{c}$ [1]. Surprisingly, in three dimensions, $k>12$ even though $k_{s}=k_{c}=12$.

Received March 1, 1994.

G. Kuperberg is supported by NSF Postdoctoral Fellowship, grant \#DMS-9107908.

O. Schramm is incumbent of the William Z. and Eda Bess Novick Career Development Chair, and is supported by NSF grant \#DMS-9112150. 
Remark 1. No packing $P$ achieves the supremum $k=k(P)$, because if $P^{\prime}$ is a translate of $P$ that meets $P$ in only one point, then $k\left(P \cup P^{\prime}\right)>k(P)$.

Let $P=\left(P_{v}, v \in V\right)$ be a packing, where $V$ is some indexing set. The nerve of $P$ is a combinatorial object that encodes the combinatorics of the packing. It is the (abstract) graph $G=(V, E)$ on $V$, where an edge $\{u, w\}$ appears in $E$ precisely when $P_{u}$ and $P_{w}$ intersect. If $P$ is a packing of round disks in the plane, then it is easy to see that $G$ is a planar graph. Conversely, the circle packing theorem [3] states that every finite planar graph is the nerve of some disk packing in the plane. This non-trivial theorem has received much attention lately, mostly because of its surprising relation with complex analysis. (Compare references [7], [5], and $[8]$.

Since the nerves of planar disk packings are understood, it is natural to ask for a description of all graphs that are nerves of ball packings in $\mathbb{R}^{3}$. In lieu of a complete characterization, which is probably intractable, Theorem 1 gives a necessary condition on such graphs: $2|E|<(8+4 \sqrt{3})|V|$.

We wish to thank Gil Kalai for a discussion which led to the question of estimating $k$.

\section{The upper bound}

Theorem 2. If $P$ is a finite ball packing in $\mathbb{R}^{3}$, then $k(P)<8+4 \sqrt{3}$.

As a warm-up, we will show that $k(P) \leq 24$. Let $E$ be the set of unordered pairs of balls in $P$ that kiss. Let $r(B)$ be the radius of a ball $B \in P$. By a famous result $([6],[4])$, it is impossible for more than 12 unit balls with disjoint interiors to kiss a unit ball $B$. If $C$ kisses $B$ and $r(C)>1=r(B)$, then $C$ contains a (unique) unit ball that kisses $B$. Thus, in a packing, $B$ cannot kiss more than 12 balls at least as large as $B$. Consider a function $f: E \rightarrow P$ that assigns to $\{B, C\} \in E$ the smaller of the balls $B$ and $C$, or either if they are the same size. Since $f$ is at most 12 to $1,|E| \leq 12|P|$. Consequently, $k(P)=2|E| /|P| \leq 24$.

The proof of Theorem 2 is a refinement of this argument.

Proof. In addition to the above notation, we let $E(B)$ denote the set of $C \in P$ such that $\{B, C\} \in E$.

Let $\rho>1$ be a constant to be determined below. For each ball $B \in P$, let $S(B)$ be the concentric spherical shell with radius $\operatorname{\rho r}(B)$. For each $B, C \in P$, define

$$
a(B, C)=\frac{\operatorname{area}(C \cap S(B))}{\operatorname{area}(S(B))} .
$$


Since the interiors of the balls in $P$ are disjoint, for any $B$,

$$
1 \geq \sum_{C \in P} a(B, C) \geq \sum_{C \in E(B)} a(B, C) .
$$

Summing over $B$,

$$
|P| \geq \sum_{\{B, C\} \in E}(a(B, C)+a(C, B)) .
$$

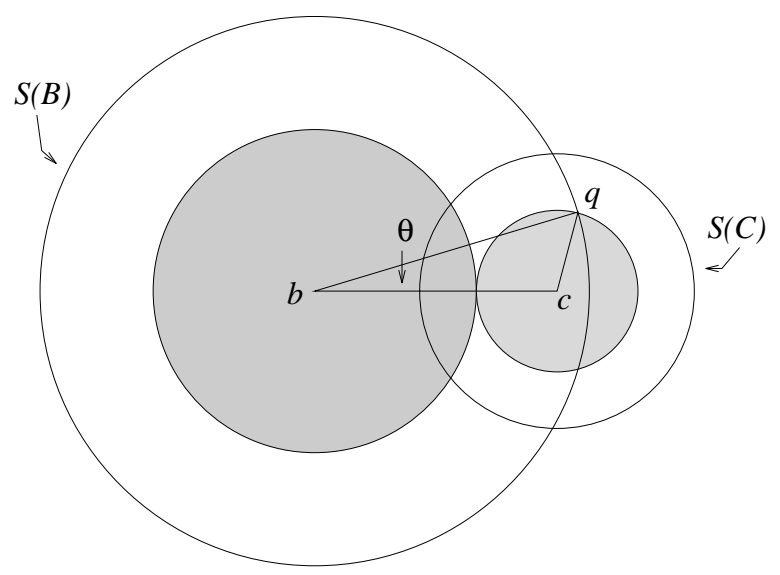

Figure 1 . The intersection of $B$ and $C$ with a plane passing through their centers.

We will obtain a lower bound on $a(B, C)+a(C, B)$ for two kissing balls $B$ and $C$. Suppose that $B$ intersects $S(C)$ and $C$ intersects $S(B)$, as shown in Figure 1. Let $b$ and $c$ be the centers of $B$ and $C$. Let $q$ be a point on the relative boundary in $S(B)$ of the spherical disk $C \cap S(B)$. Clearly,

$$
\begin{aligned}
& d(b, c)=r(B)+r(C) \\
& d(b, q)=\rho r(B) \\
& d(c, q)=r(C),
\end{aligned}
$$

where $d(x, y)$ is the distance from $x$ to $y$. Let $\theta=\angle c b q$ be the angular radius of $C \cap S(B)$. By the law of cosines,

$$
\cos \theta=\frac{(r(B)+r(C))^{2}+(\rho r(B))^{2}-r(C)^{2}}{2(r(B)+r(C)) \rho r(B)}=\frac{r(B)+\rho^{2} r(B)+2 r(C)}{2 \rho(r(B)+r(C))} .
$$

Also,

$$
\operatorname{area}(C \cap S(B))=\frac{1-\cos \theta}{2} \operatorname{area}(S(B)) .
$$


Combining equations (1), (4) and (5),

$$
a(B, C)=\frac{1}{2}-\frac{r(B)+\rho^{2} r(B)+2 r(C)}{4 \rho(r(B)+r(C))} .
$$

Switching $B$ and $C$ and adding,

$$
a(B, C)+a(C, B)=1-\frac{3+\rho^{2}}{4 \rho} .
$$

Isn't it remarkable that $a(B, C)+a(C, B)$ does not depend on $r(B)$ and $r(C)$ ? We now choose $\rho=\sqrt{3}$ to maximize the right side of equation (7). Then $a(B, C)+a(C, B)=1-\frac{\sqrt{3}}{2}$, under the assumption that $S(B) \cap C$ and $S(C) \cap B$ are non-empty. If $S(B) \cap C=\emptyset, a(B, C)=0$, which is greater than the negative value at the right side of equation (6). As a result, $a(B, C)+a(C, B) \geq 1-\frac{\sqrt{3}}{2}$ in the general case. Applying this inequality to inequality (3) yields $|P| \geq|E|\left(1-\frac{\sqrt{3}}{2}\right)$, which gives

$$
k(P)=2|E| /|P| \leq 8+4 \sqrt{3} .
$$

In conclusion, $k \leq 8+4 \sqrt{3}$. By Remark $1, k(P)<k$, establishing Theorem 2 .

Remark 2. In fact, $k<8+4 \sqrt{3}$. Let $B \in P$. Since each ball $C \in E(B)$ that intersects $S(B)$ must have $r(C) \geq(\rho-1) r(B) / 2$, there is a finite bound for the number of balls $C \in E(B)$ such that $a(B, C)>0$. Therefore there is some $\alpha<1$ (depending on $\rho$ but not $P$ ) such that

$$
\sum_{C \in E(B)} a(B, C) \leq \alpha
$$

Using this inequality in place of inequality (2) in the above proof would multiply the upper bound by a factor of $\alpha$. A good estimate for $\alpha$ would consequently strengthen Theorem 2 .

\section{The lower bound}

Theorem 3. There exists a sequence of finite packings $\left\{P_{n}\right\}$ with

$$
\lim _{n \rightarrow \infty} k\left(P_{n}\right)=666 / 53 .
$$


Observe that all questions about nerves of ball packings and average kissing numbers are invariant under sphere-preserving transformations such as stereographic projection from the 3 -sphere $S^{3}$ to $\mathbb{R}^{3}$ and inversion in a sphere.

There exists a packing $D$ in $S^{3}$ of 120 congruent spherical balls such that each ball kisses exactly 12 others [2], or 720 kissing points in total. The existence of $D$ already implies that $k(P)>12$ for some packing $P$, because by Remark $1, k>k(D)=12$.

The proof of Theorem 3 is a refinement of this construction.

Proof. We give an explicit description of $D$. Let $S^{3}$ be the unit 3-sphere in $\mathbb{R}^{4}$ and let $\tau=\frac{1+\sqrt{5}}{2}$ be the golden ratio. Choose the centers of the balls of $D$ to be the points in the orbits of $\frac{1}{2}(\tau, 1,1 / \tau, 0), \frac{1}{2}(1,1,1,1)$, and $(1,0,0,0)$ under change of sign of any coordinate and even permutations of coordinates. The radius of each ball is $18^{\circ}$. We will need the following four properties of $D$, which can be verified using the explicit description or by other means: The 12 balls that kiss a given ball have an icosahedral arrangement with 30 mutual kissing points, the centers of two kissing balls of $D$ are $36^{\circ}$ apart, the centers of two next-nearest balls of $D$ are $60^{\circ}$ apart, and $D$ is self-antipodal. (If $X$ is a point, set of points, or set of set of points in $S^{3}$, the antipode of $X$ is given by negating all coordinates in $\mathbb{R}^{4}$ and is denoted $-X$.)

Let $B_{0} \in D$ be a ball with center $b$ and let $P_{0}=D \backslash\left\{B_{0},-B_{0}\right\}$. The packing $P_{0}$ has $720-24=696$ kissing points and 118 balls. Let $R$ be the set of 12 balls in $D$ that kiss $B_{0}$, and let $S$ be the unique sphere centered at $b$ which contains the 30 kissing points between the balls in $R$. Let $I_{S}: S^{3} \rightarrow S^{3}$ be inversion in the sphere $S$. Observe that $S$ meets the boundary of each $B \in R$ orthogonally in a circle (because, by symmetry, it is orthogonal to the boundary at each kissing point), and therefore each $B \in R$ is invariant under $I_{S}$. Let $\sigma: S^{3} \mapsto S^{3}$ be the map $\sigma(p)=I_{S}(-p)$. This map $\sigma$ contracts $S^{3} \backslash\{-b\}$ towards $b$, sends $-S$ to $S$, and preserves spheres. Because $I_{S}$ leaves each $B \in R$ invariant, $\sigma$ sends $-R$ to $R$. For each $n>0$, let

$$
P_{n}=P_{n-1} \cup \sigma^{n}\left(P_{0}\right) .
$$

We claim that the sphere $S$ does not intersect any ball in $P_{0} \backslash R$. Assuming this claim, the packing $Q=P_{0} \backslash(R \cup-R)$ lies between $-S$ and $S$, and $\sigma^{n}(Q)$ is separated from $\sigma^{n+1}(Q)$ by $\sigma^{n}(S)$. Therefore each $P_{n}$ consists of an alternation of layers

$$
-R, Q, \sigma(-R)=R, \sigma(Q), \sigma^{2}(-R), \sigma^{2}(Q), \ldots, \sigma^{n}(-R)
$$

such that each layer only intersects the two neighboring layers and intersects only in kissing points. In particular, each $P_{n}$ is a packing. Moreover, 
$P_{n+1}$ has $118-12=106$ more balls and $696-30=666$ more kissing points than $P_{n}$ does. Therefore

$$
\lim _{n \rightarrow \infty} k\left(P_{n}\right)=2 \frac{666}{106}=\frac{666}{53} .
$$

It remains to check the claim. Let $B_{1}, B_{2}$ be two kissing balls in $R$. Let $b_{1}$ and $b_{2}$ be their centers and let $p$ be their kissing point. Evidently the angular radius of $S$ is $\angle b 0 p$. Using the inclusion $S^{3} \subset \mathbb{R}^{4}$ and the notation of vector calculus,

$$
\begin{gathered}
b_{1} \cdot b_{2}=b \cdot b_{1}=b \cdot b_{2}=\tau / 2, \\
b \cdot b=b_{1} \cdot b_{1}=b_{2} \cdot b_{2}=1, \\
p=\frac{b_{1}+b_{2}}{\left|b_{1}+b_{2}\right|} \\
\angle b 0 p=\cos ^{-1}\left(\frac{b \cdot\left(b_{1}+b_{2}\right)}{\left|b_{1}+b_{2}\right|}\right)=\cos ^{-1}\left(\sqrt{\frac{2+\tau}{5}}\right) \approx 31.717^{\circ} .
\end{gathered}
$$

On the other hand, the center of a ball in $P_{0}$ which is not in $R$ is at least $60^{\circ}$ away from $b$, and therefore the closest point of any such ball is at least $42^{\circ}$ away from $b$. Thus, $S$ does not intersect any such ball.

\section{References}

1. J. H. Conway and N. J. A. Sloane, Sphere Packings, Lattices, and Groups. SpringerVerlag, New York, 1988.

2. H. S. M. Coxeter, Regular Polytopes. Methuen, London, 1948.

3. P. Koebe, Kontaktprobleme der konformen Abbildung. Berichte Verhande. Sächs. Akad. Wiss. Leipzig, Math.-Phys. Klasse 88 (1936), 141-164.

4. J. Leech, The problem of the thirteen spheres. Mathematical Gazette 40 (1956), $22-23$.

5. B. Rodin and D. Sullivan, The convergence of circle packings to the Riemann mapping. J. of Differential Geometry 26 (1987), 349-360.

6. K. Schütter and B. L. van der Waerden, Das Problem der dreizehn Kugeln. Math. Annalen 125 (1953), 325-334.

7. W. P. Thurston, The Geometry and Topology of 3-manifolds. Princeton University Notes, Princeton, New Jersey, 1982.

8. Y. Colin De Verdiére, Un principe variationnel pour les empilements de cercles. Invent. Math. 104 (1991), 644-669.

Department of Mathematics, University of Chicago, Chicago, IL 60637

E-mail address: greg@math.uchicago.edu

Weizmann Institute of Science, Rehovot 76100, IsRael

E-mail address: schramm@wisdom.weizmann.ac.il 